\title{
SATELLITE PROPULSION MODELING WITH ECOSIMPRO: COMPARISON BETWEEN SIMULATION AND GROUND TESTS
}

\author{
C. R. Koppel ${ }^{1}$, J. Moral' ${ }^{2}$, M. De Rosa ${ }^{3}$, R.P. Vara ${ }^{2}$, \\ J. Steelant ${ }^{3}$, and P. Omaly ${ }^{4}$ \\ ${ }^{1}$ KopooS Consulting Ind. \\ Paris, France \\ ${ }^{2}$ Iberespacio \\ Madrid, Spain \\ ${ }^{3}$ ESA-ESTEC \\ Noordwijk, The Netherlands \\ ${ }^{4} \mathrm{CNES}$ \\ Toulouse, France
}

This paper documents the work performed for the implementation and validation of a satellite propulsion subsystem modeling library ESPSSv2.0 (European Space Propulsion System Simulation) within the existing tool EcosimPro ${ }^{\circledR}$ and using test cases relevant for space applications. EcosimPro ${ }^{\circledR}$ is a physical simulation modeling tool that is an object-oriented visual simulation tool capable of solving various kinds of dynamic systems represented by writing equations and discrete events. It can be used to study both steady states and transients. The object oriented tool, with the propulsion library allows, for example, the user to draw (and to design at the same time) the propulsion system with components of that specific library with tanks, lines, orifices, thrusters, and tees. The user enhances the design with components from the thermal library (heaters, thermal conductance, and radiators), from the control library (analogue/digital devices), from the electric library, etc.

\section{NOMENCLATURE}

$c_{p} \quad$ Specific heat at constant pressure, $\mathrm{J} /(\mathrm{kg} \cdot \mathrm{K})$

$c_{v} \quad$ Specific heat at constant volume, $\mathrm{J} /(\mathrm{kg} \cdot \mathrm{K})$

$h \quad$ Specific enthalpy, $\mathrm{J} / \mathrm{kg}$

$k \quad$ Isothermal compressibility, $\mathrm{Pa}^{-1}$

This is an Open Access article distributed under the terms of the Creative Commons Attribution-Noncommercial License 3.0, which permits unrestricted use, distribution, and reproduction in any noncommercial medium, provided the original work is properly cited. 


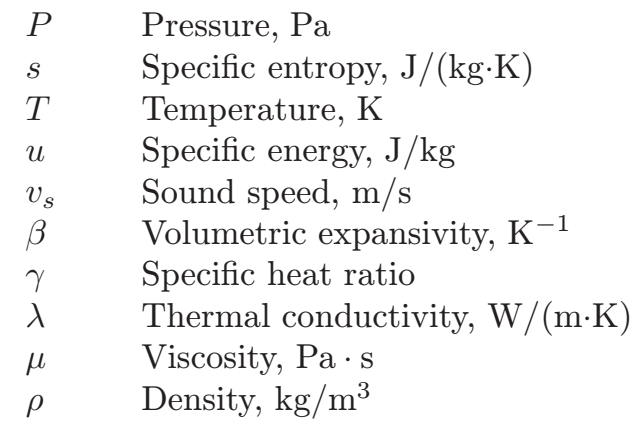

\section{INTRODUCTION}

The paper presents, first, a system engineering tool dedicated for complex systems. Second, a simulation application for designing and modeling space propulsion subsystems will be demonstrated, in particular, for the validation of the simulation results with respect to available ground experimental data, i. e., comparison of liquid filling events and gas pressurization and depressurization events.

\section{ESPSS Background}

EcosimPro ${ }^{\circledR}$ is a Physical Simulation Modeling tool developed for ESA by Empresarios Agrupados Internacional (Spain) since 1989. EcosimPro ${ }^{\circledR}$ was a precursor and now with its 20 years of careful growing, it belongs to the last generation of the common engineering tools after CAD and integrated engineering analysis tools available on classical PC.

This generation is based on a visual simulation tool for solving simple and complex physical processes that can be expressed in terms of differential-algebraic equations or ordinary differential equations and discrete events. As the tool ESPSS is relying on one-dimensional (1D) flow equations, thermodynamic relationships, and real fluid properties, there is no need for fudge factors. Therefore, the results of the simulation could be considered as general as long as the 1D flows are homogeneous monophase, or two-phase, or mixtures. Nevertheless, it is interesting to highlight in this paper some particularities between the simulation and ground tests results.

Practically, the modeling of physical components is based on a basic "EcosimPro language" (EL), an object-oriented programming language which is very similar to other conventional programming languages (Basic) but is powerful enough to write equations for modeling continuous and discrete processes. EcosimPro employs a set of libraries containing various types of components (mechanical, electrical, pneumatic, hydraulic, etc.) which can be interconnected for modeling 
complex multidomain dynamic systems. The ESA ESPSS is a set of EcosimPro ${ }^{\circledR}$ libraries written to model all aspects of a functional propulsion system.

The "Libraries" section describes those libraries, focusing on their physical modeling. Some realistic cases of interest are chosen to give an overview of software capabilities. The "Validation examples" section describes the modeling of these physical systems and comparison with experimental data.

\section{ESPSS LIBRARIES}

The following libraries have been developed as part of the 2nd phase of ESPSS: "Fluid Properties," "1D Fluid Flow," "Tanks," "Combustion Chambers," and "Turbomachinery" libraries [1,2]. An overview of these propulsion libraries is presented here.

\subsection{Fluid Properties Library}

The fluid properties library is in charge of the calculation of fluid properties. Functions available in this library are mainly used by the 1D fluid flow library for the simulation of fluid systems.

Three main classes of fluids are available:

(1) ideal gases, with temperature-dependent thermodynamic and transport properties;

(2) simplified liquids, with temperature-dependent properties; and

(3) real fluids, with tabulated properties depending on both temperature and pressure.

The last class of real fluids covers liquid, superheated, supercritical, and twophase fluids (liquid and vapor). The nomenclature lists the thermodynamic and transport properties provided for any fluid that are calculated or derived from lookup tables.

Two-phase systems and those with mixtures of two different fluids with a noncondensable (ideal) gas in any thermodynamic state are allowed. The homogeneous equilibrium model is used to calculate the properties quality (mass ratio of vapor/(vapor + fluid)), void fraction (volume ratio of vapor/(vapor + fluid)), etc.) of a real fluid in two-phase conditions, with or without a noncondensable gas mixture. Furthermore, the user can define customized fluids with tailored properties that are stored as external data files.

Hence, the fluid properties library is built upon a large external component database and consists primarily of a large collection of functions returning the value of a fluid property (or the complete thermodynamic state) by introducing 
relevant parameters. In particular, the complete set of fluid state variables (temperature, pressure, density, internal energy, enthalpy, entropy, etc.) is defined by any combination of two independent state variables (for example, density and internal energy) for a pure fluid, and three thermodynamic state variables in the case of a mixture of two fluids (for example, the two partial densities and internal energy of the mixture).

\subsection{One-Dimensional Fluid Flow Library}

The "1D fluid flow" library allows transient simulation of two-fluid, two-phase flow systems. It is linked to the fluid properties library described above, which provides the necessary functions for assessing fluid conditions. Flow inversion, inertia and high-speed phenomena are considered in pipes, volumes, and junctions. Pipes also incorporate an area-varying nonuniform mesh 1D spatial discretization into $n$ volumes. Concentrated (e.g., valves) and distributed (e.g., pipes) pressure losses are modeled, and heat transfer between metallic walls and the fluid can be taken into account. Multiple thermohydraulic correlations and initialization options are included. Other special components such as check valves, pressure regulators, heat-exchangers, and T-junctions are available.

The $1 \mathrm{D}$ pipe flows can be simulated using some of the most up-to-date, robust and accurate Computational Fluid Dynamics (CFD) techniques: centered schemes and optionally upwind schemes (P. Roe scheme). Hydraulic or pneumatic complex systems, where heat transfer and control are coupled, are easily evaluated with the $1 \mathrm{D}$ fluid flow library. Bubble formation due to the cavitation phenomena or to the presence of a noncondensable gas in a liquid is calculated in pipes or other components. Additionally, the 1D fluid flow library allows the analysis of transient phenomena due to inertia (water hammer) and bubble collapse (inertial cavitation). For hydrodynamic cavitation in terms of efficiency losses, vibrations, the 1D model represents a "first valid" approach for simulating or detecting such phenomena as long as the main flow is a $1 \mathrm{D}$ homogeneous flow, monophase or two-phase, or mixtures (with high Reynolds number, no particular two-phase boundary layer effects, etc.).

\subsubsection{Component overview}

Figure 1 shows the main library components. In an EcosimPro fluid network, every main component is either a "capacitive component" or a "resistive component:"

- a capacitive component receives the flow variables (volumetric, mass, and enthalpy) as input and returns the state variables (pressure, density, velocity, chemical composition, and enthalpy) as output; and 


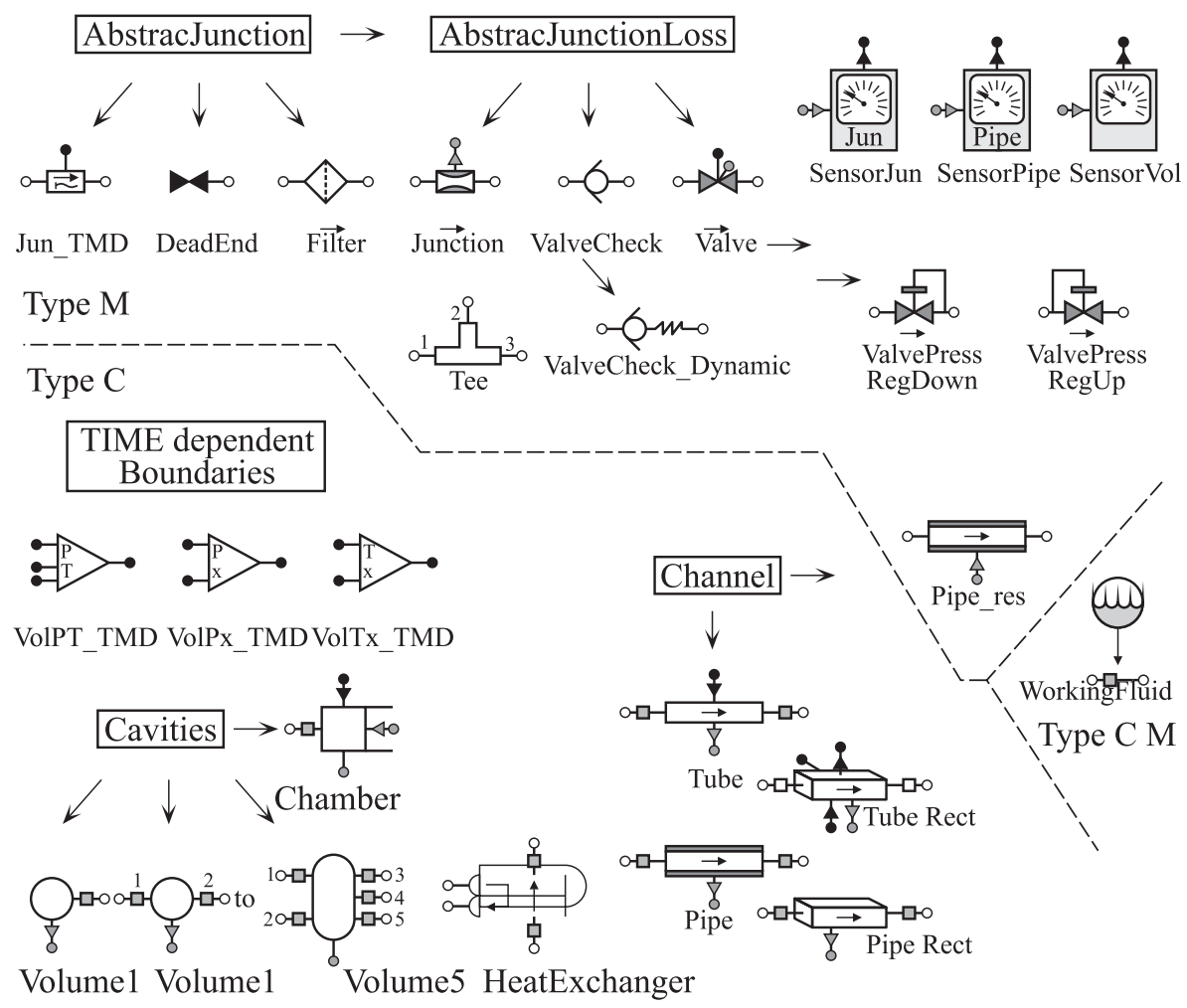

Figure 1 Symbol palette for 1D Fluid Flow Library

- a momentum resistive component receives the state variables as input for both the inlet and outlet and returns the flow variables as output.

To build a fluid network, the user has to alternately connect resistive components to capacitive ones. So, from a computational point of view, components are divided into two classes:

(1) C (capacitive) elements, integrating the mass conservation equation and the energy conservation equation. Thermodynamic functions are used to calculate the complete thermodynamic state; and

(2) M (momentum) elements, calculating explicitly the mass flows between $\mathrm{C}$ elements. It is to be highlighted that reverse flows are allowed.

Some components, like the working fluid component, have one port of type $\mathrm{C}$ and one port of type M, so that they can be connected everywhere within the circuit. 
In order to optimize the calculation time, the computational scheme prevents the occurrence of algebraic loops and high index DAE (Differential Algebraic Equations) in the mathematical model of the pipe network.

\subsubsection{Pipe component}

As an example, a detailed description of the pipe modeling is given. This component simulates an area-varying nonuniform-mesh 1D pipe that exchanges heat with a $1 \mathrm{D}$ thermal port. The general case of a mixture of two-fluid components, for which the first one can be either one- or two-phase, and the second one is a noncondensable gas, is simulated through the solution of the following 4 equations (mass conservation equations $[\mathrm{kg} /(\mathrm{m} \cdot \mathrm{s})]$, 1D Navier-Stokes $\left[\mathrm{kg} / \mathrm{s}^{2}\right]$, and energy conservation equation $\left.\left[\mathrm{kg} \cdot \mathrm{m} / \mathrm{s}^{3}\right]\right)$ :

$$
\frac{\partial \omega}{\partial t}+\frac{\partial f(\omega)}{\partial x}=\Omega(\omega)
$$

with

$$
\begin{gathered}
\omega=A\left(\begin{array}{c}
\rho \\
\rho x^{\mathrm{nc}} \\
\rho v \\
\rho u_{T}
\end{array}\right) ; \quad f(\omega)=A\left(\begin{array}{c}
\rho v \\
\rho x^{\mathrm{nc}} v \\
\rho v^{2}+p \\
\rho v\left(u_{T}+\frac{p}{\rho}\right)
\end{array}\right) ; \\
\Omega(\omega)=\left(\begin{array}{c}
0 \\
0 \\
-0.5 \rho v|v| A \frac{\Delta \zeta}{\Delta x}-\rho g A+p \frac{\Delta A}{\Delta x} \\
\frac{\Delta Q}{\Delta x}+\rho g v A
\end{array}\right)
\end{gathered}
$$

where $A$ is the front flow area; $v$ is the local velocity; $x^{\text {nc }}$ is the noncondensable mass fraction in the total mass; and $u_{T}$ is the total specific internal energy defined as $u_{T}=u+1 / 2 v^{2}$.

The set of Eqs. (1) is closed by the thermodynamic equation of state (EoS), which is described in the fluid properties library, and hereafter, written in the following general forms: $p=p(\rho, u)$, and in the case of two fluids, three state variables are required to calculate the rest of thermodynamic variables: $p$ $=p\left(\rho, x^{\mathrm{nc}}, u\right)$. In the latter case, the choice of density $\rho, x^{\mathrm{nc}}$, and internal energy $u$ as independent thermodynamic variables is the most efficient one regarding CPU-time when the EoS is taken in an arbitrary form. 
The different source terms in $\Omega(\omega)$ are the following:

- in the third equation governing the mixture momentum conservation (1D Navier-Stokes), the source term represents the equivalent distributed friction: $\Delta \zeta$ (per control volume length $\Delta x$ ), that is the pressure drop coefficient;

- in the same equation, another term accounts for gravity $g$ and the last one is responsible for the front area variation $\Delta A$ (per control volume length $\Delta x)$; and

- in the last equation governing the mixture energy conservation a thermal power source term $\Delta Q$ (per control volume length $\Delta x$ ) takes into account the heat exchange with the wall surface through a heat port and the local gravitational energy.

The implementation of this set of equations must take into account various parameters, i.e., geometry (front flow area $A$ and $\Delta x$ can vary along the pipe), numerical scheme, boundary conditions, and flow thermodynamics (state law, composition, etc.).

\subsubsection{Numerical schemes}

Either a centered or upwind numerical scheme is used to split the tube component into discrete volumes/nodes. Figure 2 describes the pipe discretization details.

The inner fluxes are computed using either a centred or upwind numerical scheme. The first and last junctions, numbered 1 and $n+1$, are the fluid ports and are defined as resistive type components using the momentum equation with sonic flow limitation. Note that the first and last half-nodal inertias are included in the junction component equations. The centered numerical scheme applies a staggered mesh approach where the state variables (pressure, density, velocity, chemical composition, and enthalpy) are associated with $n$ nodes of

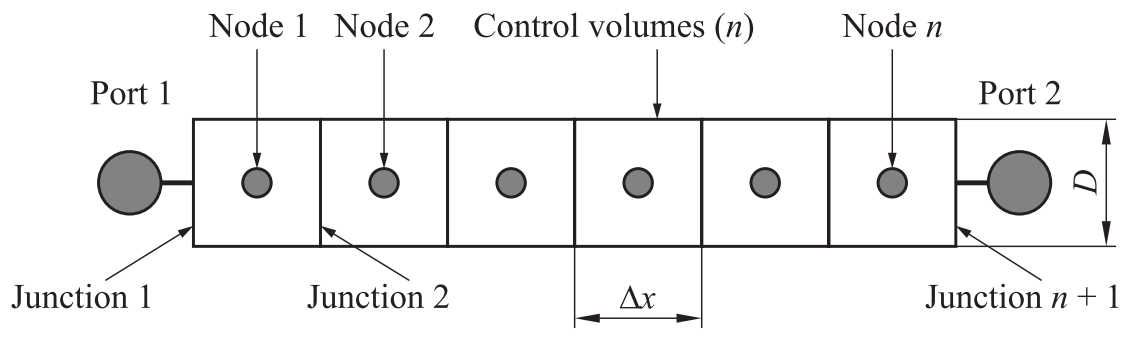

Figure 2 Pipe discretization 
control volumes, and the flow variables (volumetric, mass, and enthalpy) are calculated at the junctions of each control volumes (each junction has two half volume inertias associated with it). With the centered numerical scheme, the various fluxes to be computed in $n+1$ junctions are simply the flow variables except for the mixture momentum flux that is associated to $n$ nodes. Here, the momentum flux term includes an artificial dissipation term, "Damp," which reduces the numerical oscillations within the calculation process.

\subsection{Tanks Library}

As in the 1D fluid flow library, gas, liquid, and two-phase flow regimes can be modeled inside a Tank for ideal or real fluids. Different tank components representing the same physical component (and having formulations with different degrees of sophistication) are available in the Library: homogeneous equilibrium tank (represented by a unique temperature), tanks with two zones (gas and liquid tanks), and $1 \mathrm{D}$ tanks with $n_{\text {liq }}$ nodes in the liquid zone and $n_{g}$ nodes in the gas zone. Most typical wall shapes have been included as separated components to model heat conductivity in walls and insulations. Figure 3 shows the main library components.

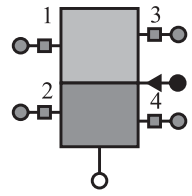

Tank_Single

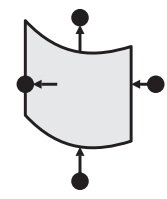

Cylinder_ins



Tank_CylDomes

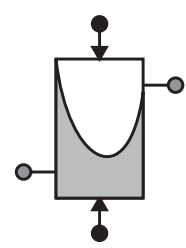

Tank_Bladder

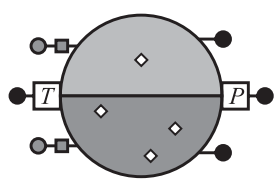

Tank_Sphere

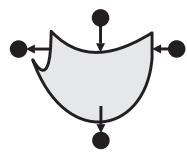

Sphere_ins

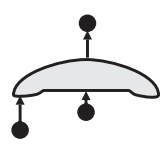

Dome_ins



Tank_CylDomesSph

Figure 3 Symbol palette for Tanks Library

\subsection{Turbomachinery Library}

Turbomachinery is an EcosimPro ${ }^{\circledR}$ library for the simulation of pumps, turbines, and compressors. Pump components are provided with dimensionless turbo- 


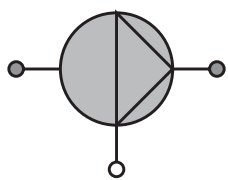

Pump

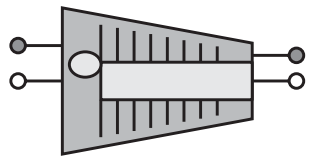

Compressor 1

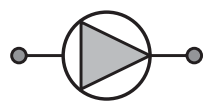

Pump_vacuum

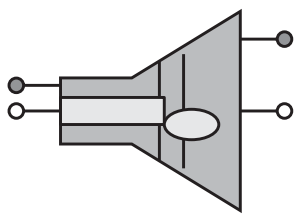

Turbine1

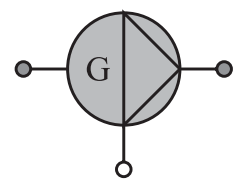

Pump_gen

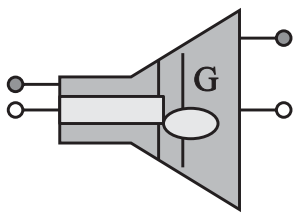

Turbine_gen 1

Figure 4 Symbol palette for Turbomachinery Library

pump characteristics curves adapted to positive and negative speeds and flow zones. Turbine and compressor components are provided with dimensionless performance maps as a function of the reduced axial speed and pressure ratio. Figure 4 shows the main library components.

\subsection{Combustion Chambers Library}

The combustion chambers library enables the simulation of rocket engines and thrust chamber elements. Combustion gas mixture properties (transport and specific heat) are calculated from adequate coefficients from each chemical species present as a combustion product. Minimization of Gibbs free energy is applied to find equilibrium molar fractions for a mixture of reactants. Figure 5 shows an overview of the combustion chambers components.

The 1D combustor components have the main advantage of being able to simulate startup and shutdown sequences. They can be directly connected to the respective pipes, valves, and regenerative circuits of a real-like engine system. In this respect, many cases have been run successfully for modeling different kinds of engine cycle (gas generator cycle, staggered cycle, and expander cycle) where the turbomachinery and feeding tanks are included.

\subsection{Other Compatible Libraries}

Three of the already existing libraries within the EcosimPro software are compatible with the ESPSS libraries and their components can be used in combination with the ESPSS components. These libraries are as follows. 

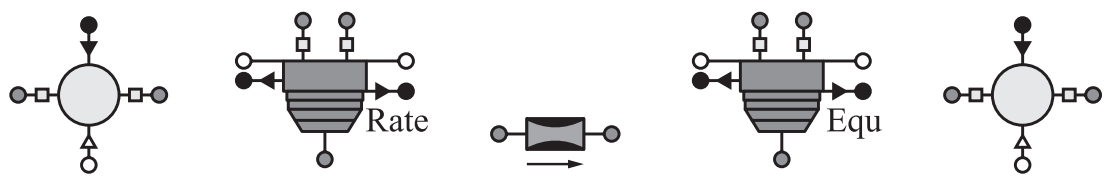

Inj_Cavity_rate Combustor_rate
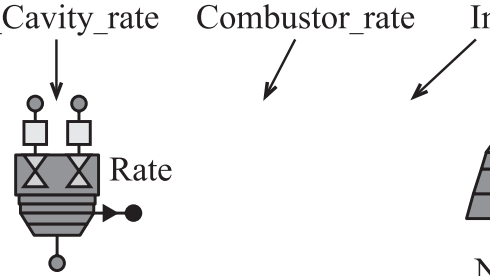

Injector

Combustor_eq

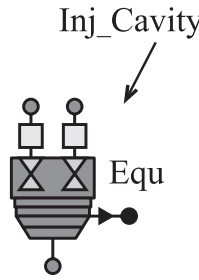

PreBurner_rate
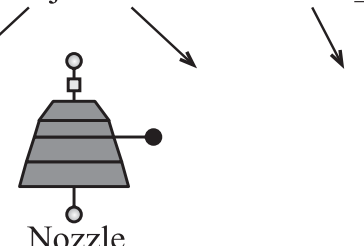

Cavity
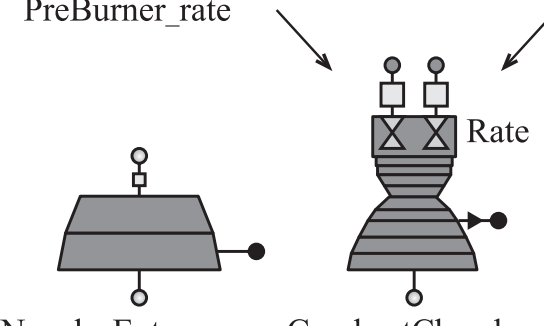

Nozzle_Ext

CombustChamber

Nozzle rate

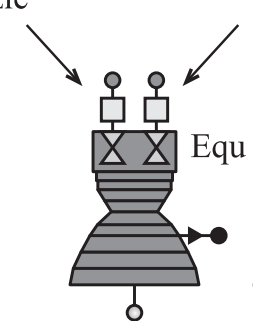

CombustChamber
Nozzle eq Circuit
PreBurner_eq

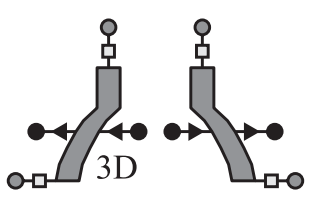

Cooling

Jacket

Figure 5 Symbol palette for Combustion Chambers Library

\subsubsection{Thermal Library}

This library provides components for the prediction of temperature distributions and heat fluxes in systems and devices using the thermal network method. The thermal network method is also known as the lumped parameter method or the resistance/capacity method. It is essentially a finite difference method and involves modeling a continuous medium as the discrete thermal network of nodes representing the capacitance of the system linked by conductors representing its conductance. The modes of heat transfer that can be considered in thermal model are conduction, radiation, and natural convection. Additional features allow the handling of phase-change phenomena using components called "DNphases."

\subsubsection{Mechanical Library}

The mechanical library allows the modeling of 1D mechanical systems. Most of the typical mechanical units are included as components that can be used to build graphically complex systems: 
- translational components for systems with linear displacements driven by forces with frictional losses;

- rotational components for systems with angular displacements driven by momentum with frictional losses; and

- kinetic converters (levers, gears, ideal rotational-to-translational gear) transforming an angular movement into other angular movement at different velocity or into a translational movement.

The most important elements can be grouped in the following groups: masses and inertia (Sliding mass, End stop mass, Inertia and End stop Inertia), which implement the second Newton law. Force and torque generators (Coulomb friction, dampers, and springs) calculate force or torque from the position or the velocity in their ports. Actuators (force, position, torque, acceleration, and angle generators) provide force, torque, acceleration, position, and angle depending on an external input signal (user defined law).

\subsubsection{Control Library}

This library provides the custom items needed to represent analogue and digital control systems. All basic operations can be performed on analogue or digital signals (addition, multiplication, multiplex/demultiplex, derivative, integral, logical operations), and some more advanced control operations are implemented (linear or discrete space-state, filter, PID control, transfer functions, etc.).

\section{VALIDATION CASE: FILLING OF EMPTY TUBES}

The filling of an empty tube from a pressurized tank (at a pressure $P_{\text {tank }}$ ), induces at the end of filling a first water-hammer peak pressure $(\Delta P)$ followed by, after a rather long duration ( $\Delta$ Time), a second water hammer peak having lower amplitude $\left(\Delta P_{2}\right)$. This is very similar to an elastic bouncing ball. This typical behavior can be assessed for the first peak pressure according to Joukowsky, cited in [3] and for the duration between peak pressures according to Wylie and Streeter, cited in [3] with the two following simple equations (see the sketch in Fig. 6):

$$
\begin{aligned}
\Delta P & =v_{s} \rho v ; \\
\Delta \text { Time } & =\frac{\Delta P}{P_{\text {tank }}} \frac{2 L}{v_{s}}
\end{aligned}
$$

where $v$ is the velocity of fluid; $L$ is the empty tube length; and $v_{s}$ is the speed of sound. 


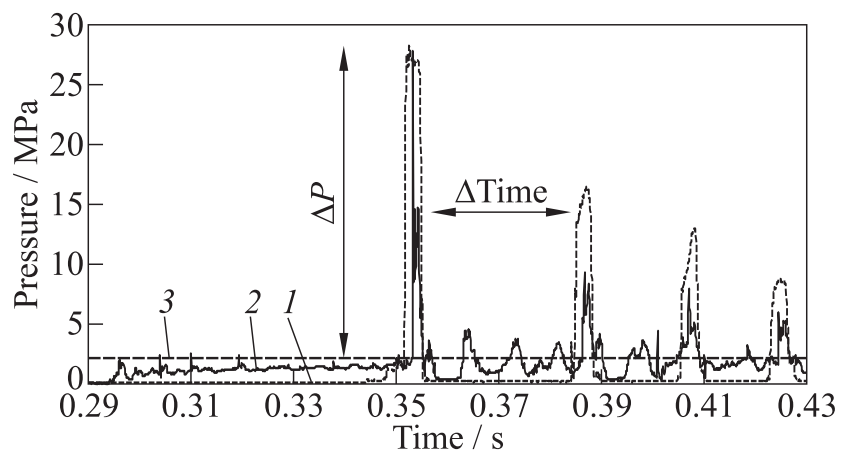

Figure 6 Water hammer pressures from ground tests: 1 - downstream pressure $P_{d}$; 2 - upstream pressure $P_{u}$; and $3-P_{\text {tank }}$

There are no simple equations for the assessment of the amplitude of $\Delta P_{2}$, except that the damping, due to the friction on the tube walls, allows a reduction of the velocity $v$; hence, one can only state that $\Delta P_{2}<\Delta P$.

In order to validate the computations performed by the tool EcosimPro ${ }^{\circledR}$ with the equations set into the ESPSS libraries, the tests data from existing

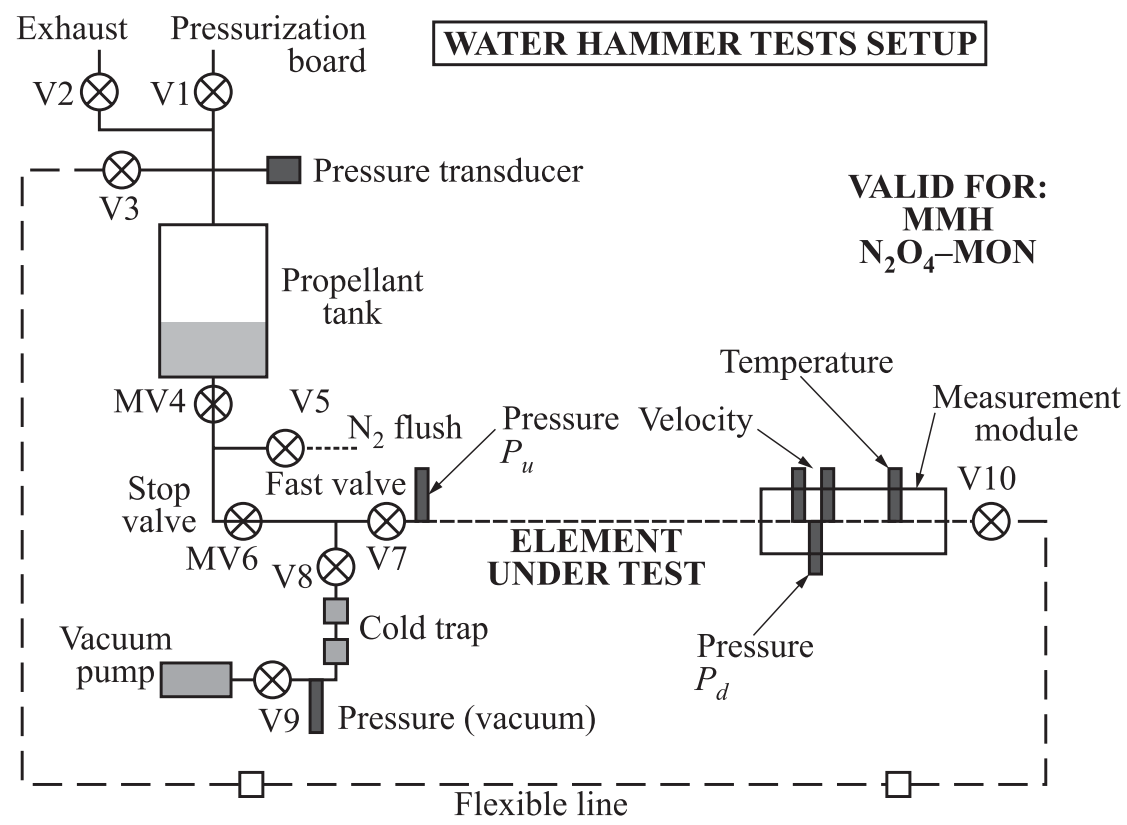

Figure 7 Setup for water hammer tests (Onera) 


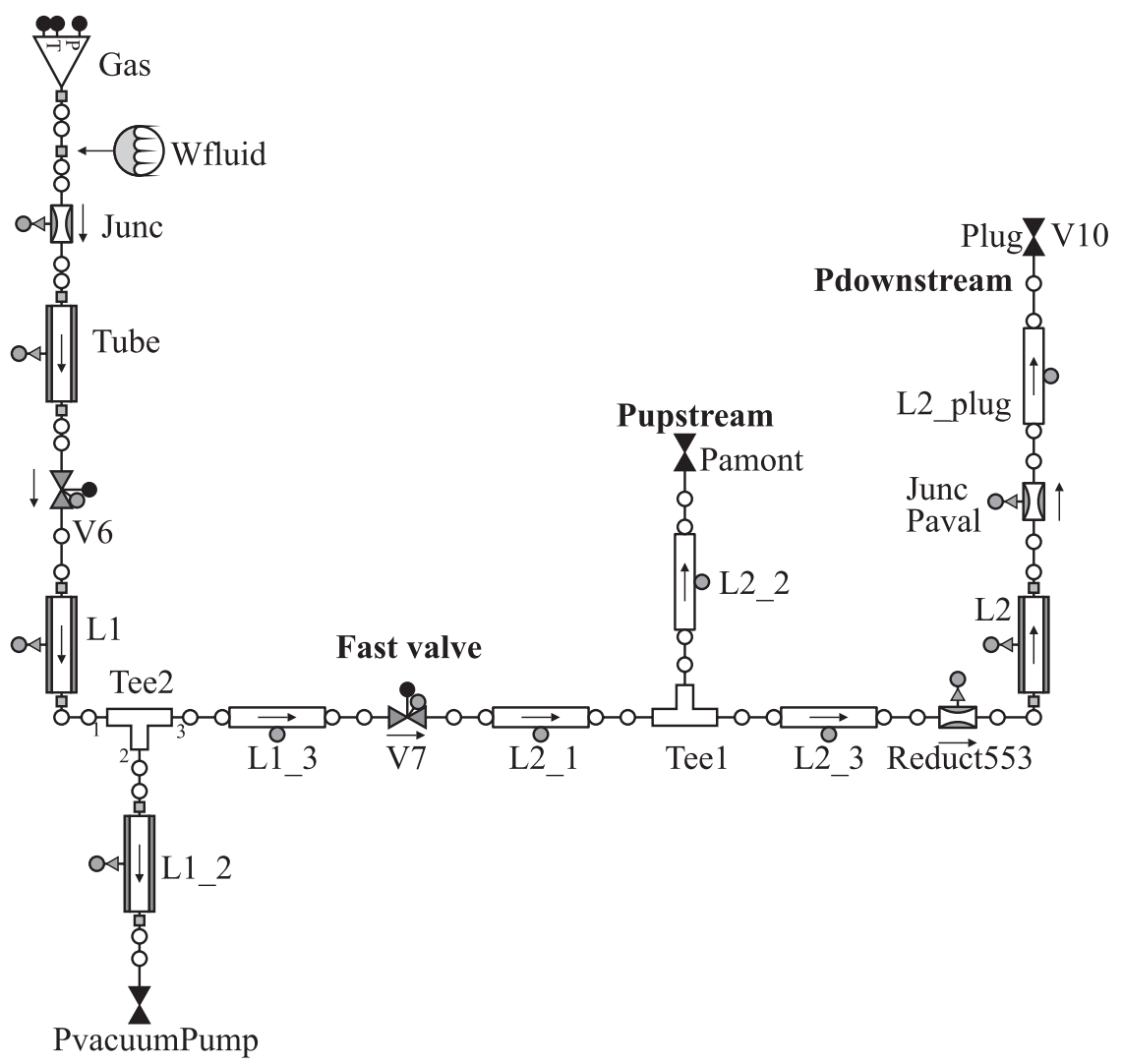

Figure 8 Test setup for water hammer under EcosimPro ${ }^{\circledR}$ with objects of ESPSS

ground experiments performed by Onera Le Fauga and provided by CNES [4] have been compared to the results of the modeling.

The test setup, described in [4-6], is defined by a line filled up to the fast valve V7 that isolates the empty line up to the valve V10. Two main pressure measurements are available upstream, $P_{u}$, near V7, and downstream, $P_{d}$, near V10. A model representing this test setup has been created using the generic objects of the ESPSSv2.0 library [7] that have been set to have the specific dimensions of the hardware. The schematics of the hardware test setup and the EcosimPro ${ }^{\circledR}$ model are shown in Figs. 7 and 8.

The pressure outputs of the ground test with water are shown in Fig. 9 as well as the results of EcosimPro and ESPSS simulation results.

As shown in Fig. 9, the results of the simulation (without use of any fudge factors) are surprisingly good with respect to the experimental ground data: 

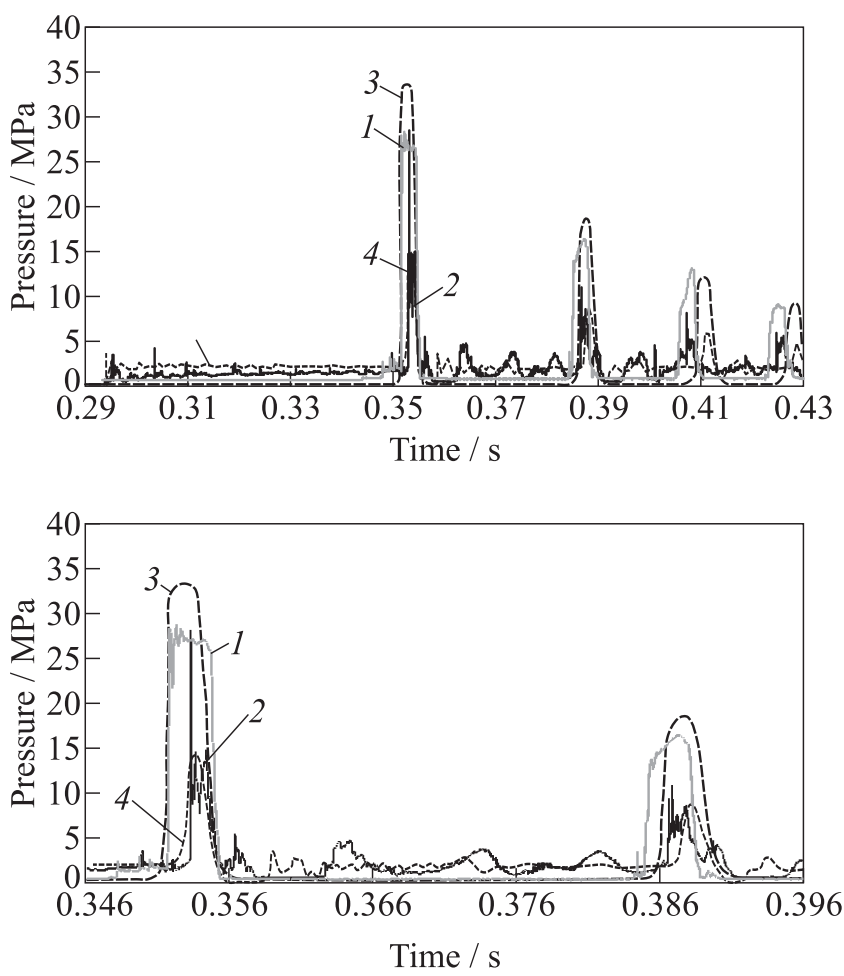

Figure 9 Comparison between water hammer pressures: simulation results are robust and safe: $1-P_{d} ; 2-P_{u}$; and 3 and $4-P_{\text {node }}$ at L2 and L2_2, respectively. Solid curves refer to test data and dashed curves to EcosimPro simulation. Lower plot shows the exploded view of the upper plot

very similar peak pressures for the first peak pressure (a bit higher for the simulation: that is a quality of robustness of the simulation for being used safely for designing and for the justification of the design): this robustness has always been observed in the course of the program, but for keeping a prudent rule, it cannot be assured that this robustness is valid for any other simulation, especially when important parameters of the test (like the fluid temperature, for example) are not well taken into account in the simulations. The duration between the two first spikes is also nearly identical and the damping for the second pressure peak is also very compliant with the experimental data.

This particular result with water has been confirmed with several other fluids for which ground test data were available ( $\mathrm{MMH}, \mathrm{N}_{2} \mathrm{O}_{4}, \mathrm{~N}_{2} \mathrm{H}_{4}$ ). 


\section{VALIDATION: FILLING A SATELLITE MANIFOLD}

A similar comparison between the modeling of the water hammer phenomenon occurring in a satellite propulsion system with actual test data has also been carried out. Here, the test data have been provided by CNES from the Stentor satellite propulsion system functional model testing [8].

Figure 10 shows the Stentor satellite propulsion system functional model used for the ground tests. This functional model represents only a certain section of the propulsion system as illustrated in Fig. 11 with the location of the pressure transducers (modules). However, the configuration of the functional model is



Figure 10 Satellite hardware

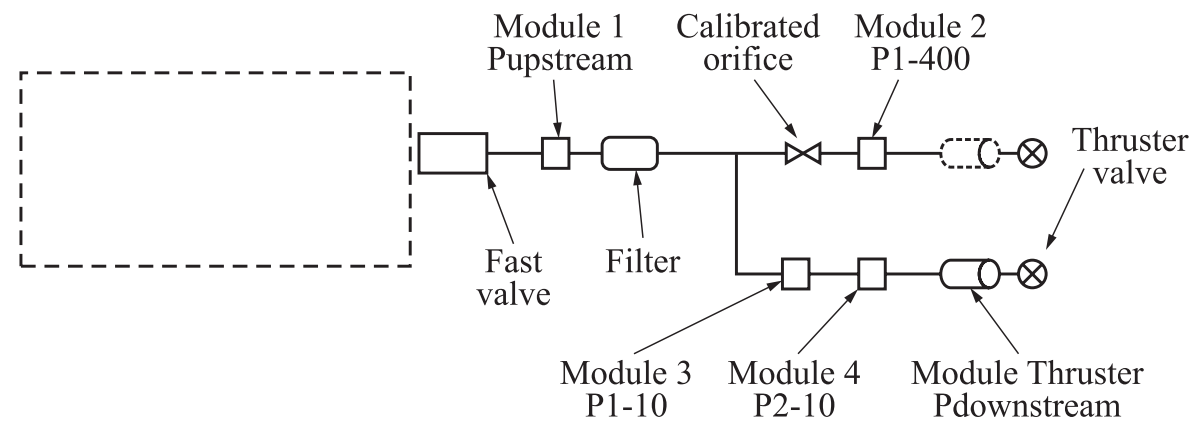

Figure 11 Sketch of the tee tubing to be filled (from Onera document [8]) 


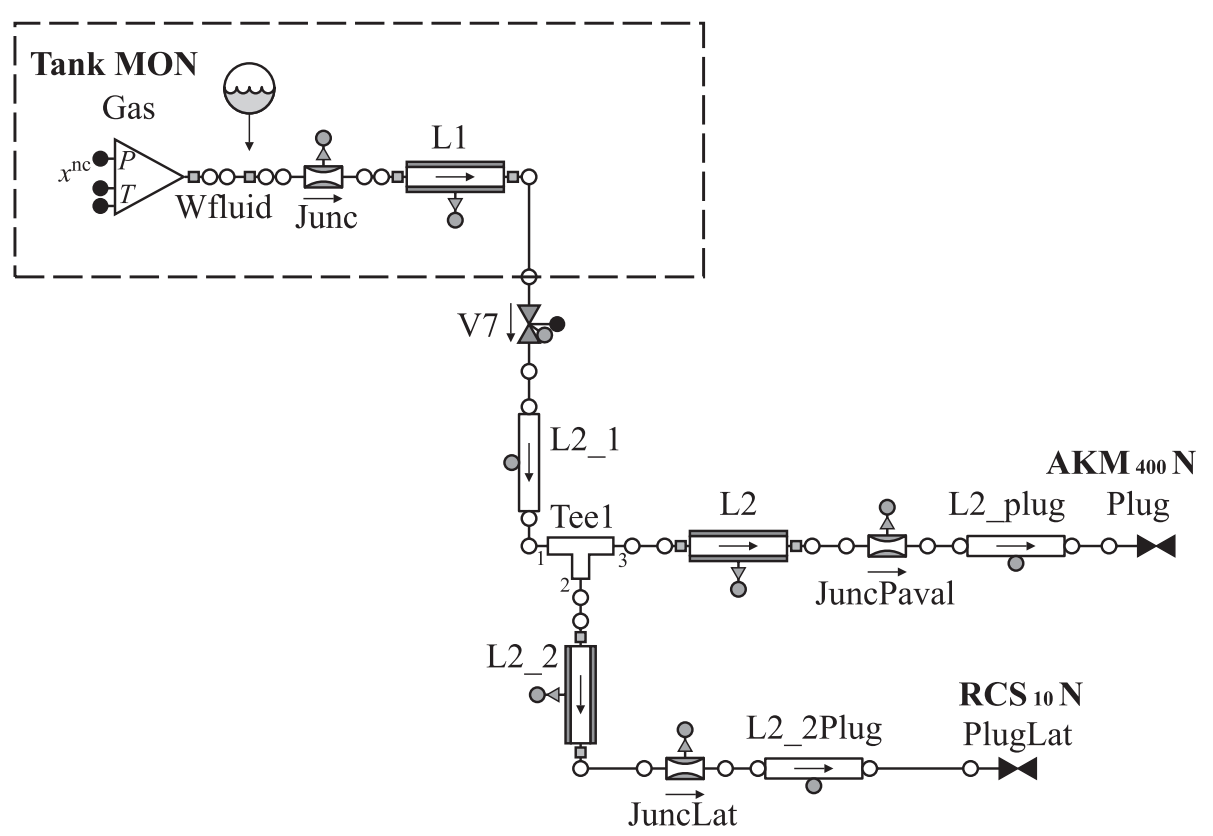

Figure 12 EcosimPro ${ }^{\circledR}$ model with ESPSS objects (the calibrated orifice is included into the "Tee1" data)

represented with titanium pipe, all bend pipes and equipment locations within this section on the flight model.

Figure 12 shows the EcosimPro/ESPSS model used for the simulation. It should be noted that the modeling of the LAE (liquid apogee engine) orifice, which is implemented in the hardware on the LAE branch just downstream the tee junction, was actually integrated into the component Tee1 modeling.

Also of note is that the filter used in the hardware was not included in this EcosimPro/ESPSS model. A filter can introduce additional friction in the fluid, and due to its internal volume, it can introduce a delay in the propagation of water hammer waves. This exclusion of the filter from the model was considered acceptable because its small internal volume produced a second-order effect on the system behavior.

As shown in Fig. 13, the results of the simulation (without use of any fudge factors) are already surprisingly similar to the experimental data with water, with a safe robustness for the simulated pressures that are always higher than the pressures obtained in experimental tests.

Figure $13 b$ shows the details of the first two pressure peaks. As can be seen, the simulated pressure spikes within both the LAE and RCT (reaction 


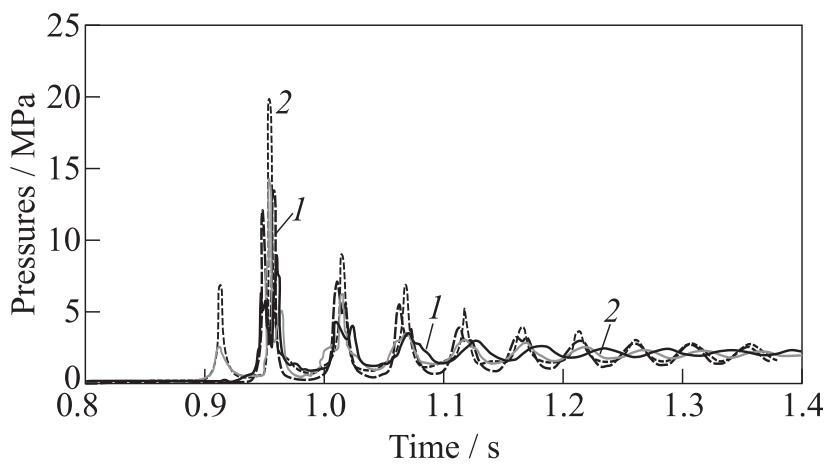

(a)

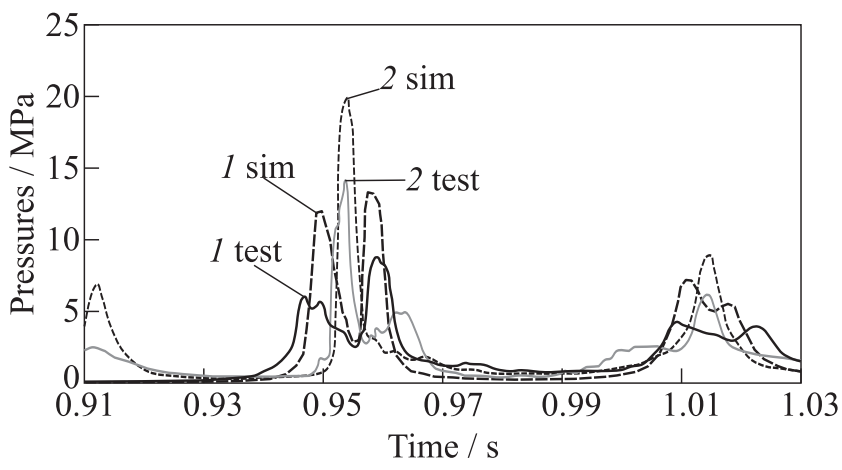

(b)

Figure 13 Comparison of water hammer pressures: $1-P_{\mathrm{av}}=10 \mathrm{~N}$; and $2-P_{\mathrm{av}}$ $=400 \mathrm{~N}$. Solid curves refer to test data and dashed curves to EcosimPro simulation. Lower plot shows the exploded view of the upper plot

control thruster) lines are in-line with the test data thereby demonstrating the consistency between the model and the hardware. This consistency has been demonstrated relying only on the equations used in the library and on the specific fluid properties, without using any fudge factor; thus, this consistency can be considered, at first, as general for any fluid.

\section{VALIDATION CASE: BUTANE PRESSURIZATION}

The validation of the ESPSS modeling of the behavior of two-phase mixtures in the dynamic processes of pressurization and expulsion has been made possible due to available test data for butane propulsion performed by Air Liquide 


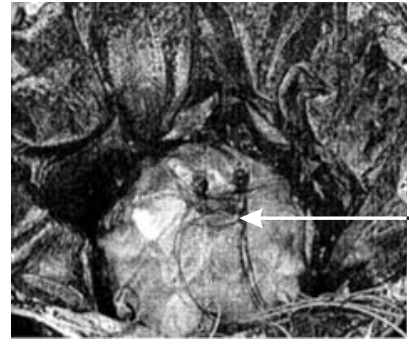

(a)

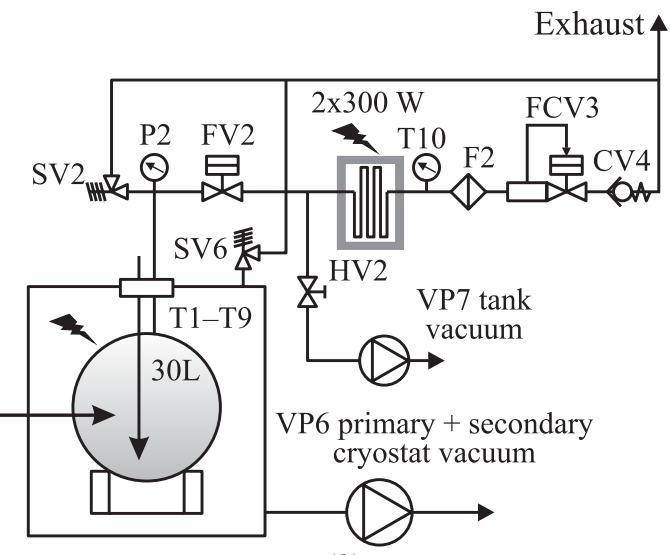

(b)

Figure 14 Insulated spherical tank (Photo Air Liquide) (a); and sketch of the experimental setup at Air Liquide (b)

DTA [9]. The schematic of the test setup is shown in Fig. $14 a$ and comprises a very well thermally-insulated spherical storage tank with heaters (Fig. 14b) connected to a series of valves and a mass flow regulator by tubing.

Many types of tests were performed with this butane cold-gas propulsion system test setup; however, the test sequence most of interest for the validation process of the ESPSS comprised of two stages. Starting from ambient conditions, there was first a long-duration heating of the tank in order to reach a significantly

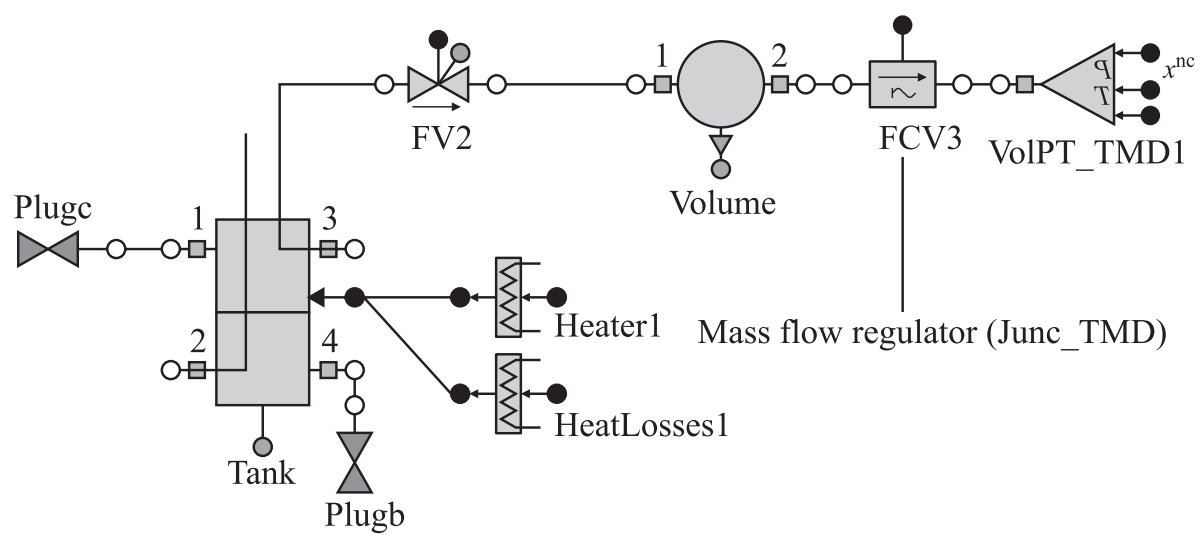

Figure 15 Test setup for butane test under EcosimPro ${ }^{\circledR}$ with ESPSS objects 


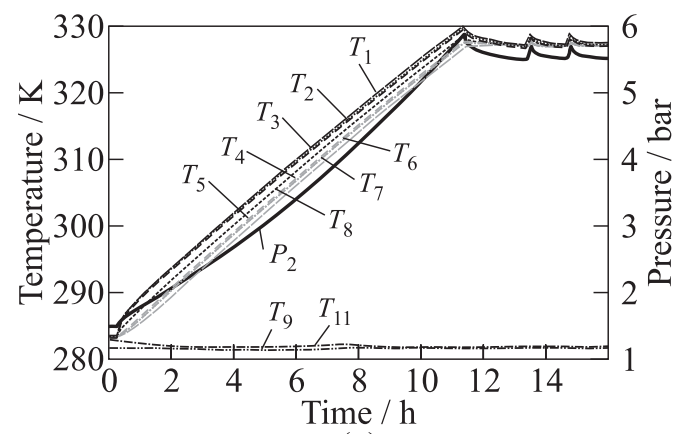

(a)

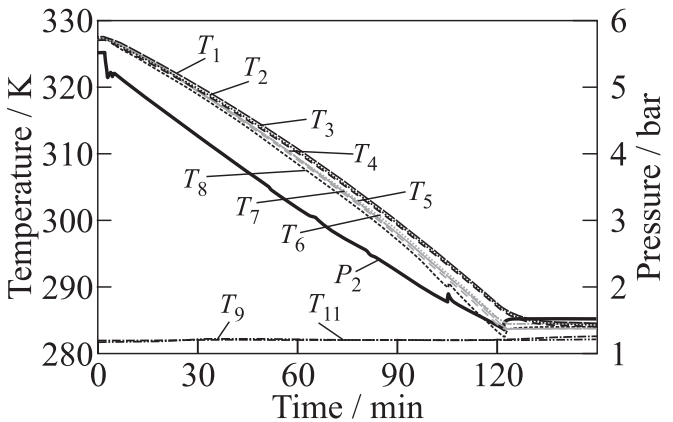

(b)

Figure 16 Results of ground tests during the pressurization and expulsion phases: several tank skin temperatures and tank pressure (Document Air Liquide [9])

high pressure followed by the expulsion of the butane gaseous phase at a constant mass flow rate in order to simulate the feeding of the thrusters.

Using the generic components of the ESPSS libraries, the EcosimPro ${ }^{\circledR}$ model of this cold-gas butane propulsion system was generated, as shown in Fig. 15. Once created, various simulations of the actual tests performed on the hardware were run using this model.

The simulation on EcosimPro ${ }^{\circledR}$ starts by the heating of the tank for a duration of $39600 \mathrm{~s}$ [9]. Then, the expulsion phase starts with the duration of $7200 \mathrm{~s}$. The results of the ground test during the pressurization and the expulsion phases are shown in Fig. 16. Note in Fig. 16b: temperatures decrease sharply from 327 by $1 \mathrm{~K}$, then slowly to $284 \pm 1 \mathrm{~K}$; pressure decreases sharply from 0.55 by $0.03 \mathrm{MPa}$, then slowly down to $0.153 \mathrm{MPa}$; duration $7200 \mathrm{~s}$. The output of the simulation tool (with the pressurization phase immediately followed by the expulsion phase) is shown in Fig. 17 (without use of any fudge factors). It is to be mentioned that the $60000 \mathrm{~s}$ of the ground test are simulated dynamically within less than one minute on a small laptop. 


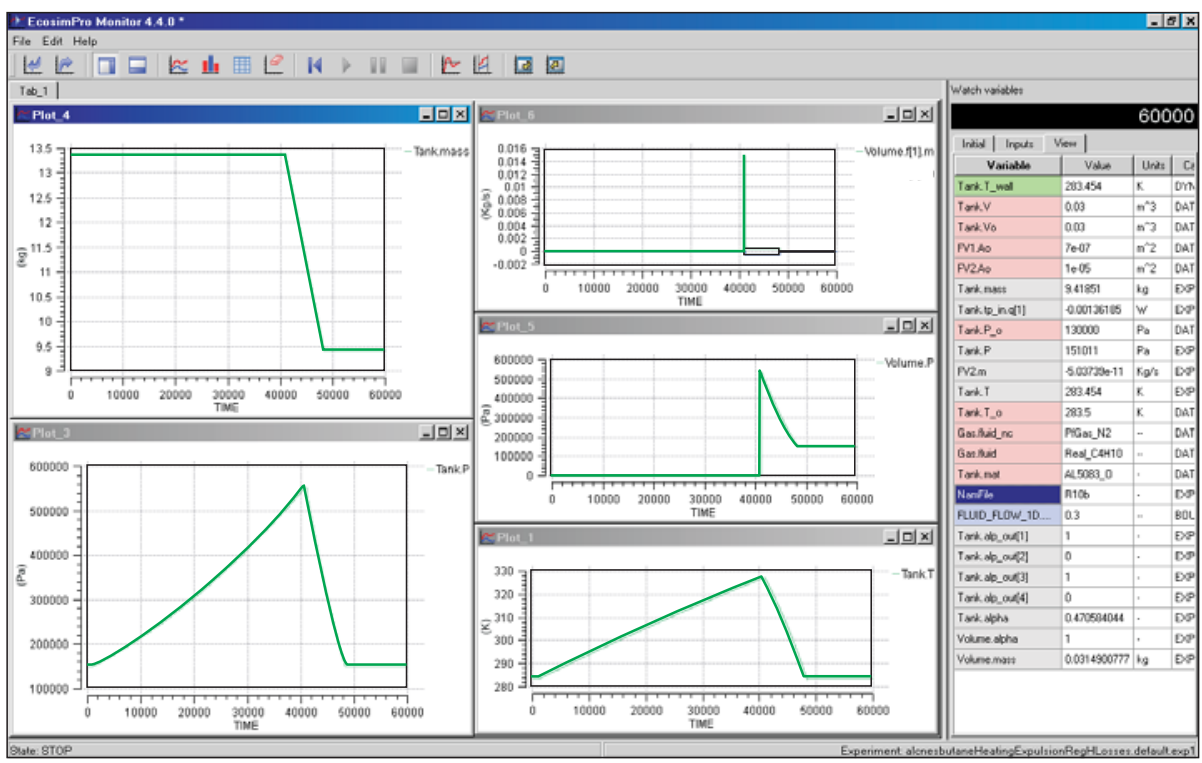

Figure 17 Output of the simulation tool up to 60000 s: mass, pressure, mass flows, and temperatures (with $n$-butane real gas). (Refer Koppel et al., p. 762.)

The quantitative results for the heating phase with the real experimental test and the simulation are shown in Table 1 . The results are very similar. This shows the suitability of the data and equation used in the ESPSS for solving the problem of heating a two-phase mixture. The quantitative results for the expulsion phase with the real experimental test and the simulation is shown in Table 2. The results are almost identical. This shows once again the suitability

Table 1 Comparison of experiments and simulation for the heating phase

\begin{tabular}{ccc}
\hline Heating (pressurization) & $\begin{array}{c}\text { Temperature initial } \\
\text { phase }\end{array}$ & $\begin{array}{c}\text { Pressure initial } \\
\text { to final, } \mathrm{K}\end{array}$ \\
\hline Ground test & 283 to 329 & 0.150 to $[0.58-0.55]$ \\
EcosimPro simulation & 283.5 to 327 & 0.151 to 0.553 \\
\hline
\end{tabular}

Table 2 Comparison of experiments and simulation for the expulsion phase

\begin{tabular}{ccc}
\hline Expulsion phase & $\begin{array}{c}\text { Temperature initial } \\
\text { to final, } \mathrm{K}\end{array}$ & $\begin{array}{c}\text { Pressure initial } \\
\text { to final, } \mathrm{MPa}\end{array}$ \\
\hline Ground test & 327 to 284 & 0.5515 to 0.153 \\
EcosimPro simulation & 327 to 283.5 & 0.5519 to 0.151 \\
\hline
\end{tabular}


of the data and equations used in the ESPSS for solving the problem of managing a two-phase mixture.

\section{CONCLUDING REMARKS}

The physical simulation modeling tool EcosimPro ${ }^{\circledR}$ combined with the ESPSS libraries has been presented and assessed for several validation cases relevant in the area of propulsion subsystems for satellite platforms. The results of the simulations show good compliances with the on-ground experimental results.

With respect to the water hammer pressure, the simulation produces slightly higher pressures than the experimental data. This robustness enables the use of the tool and library for operational purpose, for designing safely the propulsion systems with respect to the water hammer and with a drastic reduction of the tests to be performed for the justification of the design.

The main advantage of using this new generation of simulation tool is the capability of managing complex systems, subsystems, and their components like different engineering objects. There was no need to develop several software tools, one for water hammer and another for two-phase mixture: the components described by the equations in the unique set of ESPSS libraries can be used like hardware components for solving or assessing many different problems saving lots of efforts and money. In addition, a detailed analysis of simulation data can highlight the occurrence of certain conditions required for triggering particular phenomena, like so-called "adiabatic decomposition of hydrazine" during the filling of lines, etc.

EcosimPro ${ }^{\circledR}$ has been already successfully used for several space applications and in many other areas. The fidelity and quality of the simulations obtained implies that this simulation concept of new generation with ESPSS libraries will become in the next years a commonplace like CAD stations today.

\section{ACKNOWLEDGMENTS}

The authors want to express their special thanks to CNES-Toulouse for providing very valuable test data. The overall activities have been performed in the frame of an ESA/ESTEC Contract 21490/08/NL/CP.

\section{REFERENCES}

1. De Rosa, M., J. Steelant, J. Moral, Y. Elkouch, and R. P. Vara. 2008. 5th European Space Propulsion Conference (ESPSS: European Space Propulsion System Simulation). Heraklion, Crete. 
2. Koppel, C., J. Moral, M. De Rosa, R. P. Vara, J. Steelant, and P. Omaly. 2009. A Satellite platform modelling with EcosimPro: Results of the simulation compared to the ground tests. SimTecT Simulation Conference and Exhibition. Adelaide, Australia.

3. Bergant, A., A. R. Simpson, and A. S. Tijsseling. 2006. Water hammer with column separation: A historical review. J. Fluids Structures 22:135-71. Litostroj Ljubljana, University of Adelaide (Australia), Eindhoven University.

4. Maisonneuve, Y., and J Maunoury. 2005. Etude expérimentale du phénomène de coup de bélier en ergols réels. Confidentiel Onera-Cnes. RT 1/06416 DMAE Version 2.

5. Gibek, I., and Y. Maisonneuve. 2005. Waterhammer tests with real propellants. AIAA Paper No. 2005-4081.

6. Lecourt, R., and J. Steelant. 2007. Experimental investigation of waterhammer in simplified feedlines of satellite propulsion system. J. Propul. Power 23(6):1214-24.

7. Library ESPSS dated March 2008.

8. Maisonneuve, Y., and J. Maunoury. 2007. Etude expérimentale du phénomène de coup de bélier sur réseau. Confidentiel Onera-Cnes. RT 4/10570 DMAE.

9. Ravex, A., S. Bur, and P. Bravais. 2007. Rapport d'essais des essais de soutirage C4H10. Confidentiel AirLiquide-Cnes. D8014-NT-010 (0). 